\title{
Expectations for first pair-production of top-quarks in the semi-leptonic channel in CMS at $\sqrt{s}=10 \mathrm{TeV}$
}

\author{
Teh Lee Cheng (on behalf of the CMS Collaboration) ${ }^{1}$ \\ University of Bristol, $U K$ \\ E-mail: t.1.cheng@bristol.ac.uk
}

\begin{abstract}
The top quark will be a fundamental element of the early physics program at the Large Hadron Collider (LHC). Given the complex signature of this "most exotic" of all known SM particles, the pair production of top quarks will be a crucial instrument for the commissioning the LHC experiments' tools for physics analysis. Only when the first top-quark signal has been established will the experiments be able to use it to further probe the standard model, and to begin the search for new physics that the LHC is almost certain to deliver. We will discuss the plans and analysis strategies of CMS to pursue this physics program, and show the expected performance of the experiment with a focus on an early cross-section measurement in the channel where the $\mathrm{W}$ boson from one top quark decays into leptons, while the other $\mathrm{W}$ decays into quarks.
\end{abstract}

European Physical Society Europhysics Conference on High Energy Physics EPS-HEP 2009,

Krakow, Poland

July 16-22, 2009

1 Speaker 


\section{Introduction}

At the LHC, with a centre-of-mass energy of $10 \mathrm{TeV}$, about 8300 top-quark pairs will be produced with an integrated luminosity of $20 \mathrm{pb}^{-1}$. The experimental signatures for the semileptonic decay of top quark pairs are one highly energetic electron/muon, four energetic jets and missing transverse energy. We present the expectation of the CMS experiment [1] assuming 20 $\mathrm{pb}^{-1}$ of early data using robust cut-based analyses without the use of b-tagging [2-4].

\section{Expectation in the Semi-leptonic Electron Channel (e+jets)}

To select the signal event in the e+jets channel, we first require the event to pass a single electron trigger. Next we require exactly one isolated electron with at least $30 \mathrm{GeV}$ in transverse energy $\left(\mathrm{E}_{\mathrm{T}}\right)$ and within tracker acceptance (pseudorapidity $|\eta|<2.5$ ) plus some quality cuts. Events containing isolated muon are rejected. Lastly we require at least four jets with at least 30 $\mathrm{GeV}$ in transverse momentum $\left(\mathrm{p}_{\mathrm{T}}\right)$ and $|\eta|<2.4$.

To reduce backgrounds from Z+jets and QCD multijet events, two approaches have been explored. In the first approach (Option 1), secondary loose electrons are vetoed and the $\eta$ acceptance of electron is restricted to 1.442. In the second approach (Option 2), cuts on di-electron invariant mass and on missing $\mathrm{E}_{\mathrm{T}}$ of $20 \mathrm{GeV}$ are imposed, and electrons from photon conversion are vetoed. After the selection, we expect 172 (182) signal events and 108 (147) background events for $20 \mathrm{pb}^{-1}$ of data for Option 1 (2). The composition of events as a function of jet multiplicity is shown in Figure 1 for Option 2.

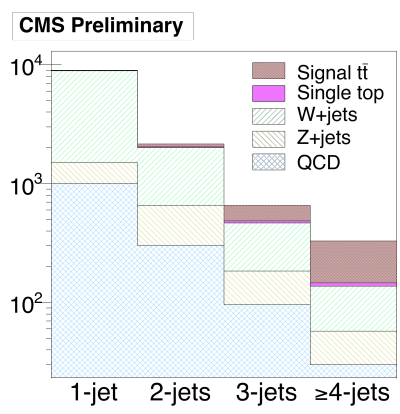

Figure 1: Event composition as a function of jet multiplicity for the e+jets channel for $20 \mathrm{pb}^{-1}$.

Fake prompt electrons from QCD multijet events can arise from charged pion/photon overlaps, heavy flavour decays, charge exchange background and converting photons. As QCD backgrounds cannot be reliably simulated, a data-driven method is developed to estimate it using an electron isolation variable, by fitting a QCD-dominated region (large isolation) and extrapolate into the signal region (low isolation).

The dominant background comes from $\mathrm{W}+$ jets events. A template fit method is used to extract the number of signal event. We used the maximum likelihood method and a discriminating variable called "M3", defined as the invariant mass of the three jets yielding the largest vectorial-sum in $\mathrm{p}_{\mathrm{T}}$. From the fitted number of signal events, the cross section can be calculated using the formula $\sigma(t \bar{t})=N_{t \bar{t}}^{f i t} /\left(A \cdot \varepsilon_{t \bar{t}} \int L d t\right)$. The statistical uncertainty on the cross section is estimated to be $23 \%$ for $20 \mathrm{pb}^{-1}$ using ensembles of pseudo-experiments, and is expected to reduce to $10 \%$ when $100 \mathrm{pb}^{-1}$ of data is collected. Uncertainty in luminosity is expected to be $10 \%$.

Important sources of systematic uncertainty on the cross section measurement has been evaluated, including jet energy scale uncertainty, signal $\mathrm{MC}$ generator, uncertainty in initial/final-state radiations in signal events, uncertainty in factorization scale and matching threshold in the $\mathrm{W}+$ jets $\mathrm{MC}$, shape uncertainty of single top and QCD, and parton distribution 
function uncertainty. The total systematic uncertainty is estimated to be $20 \%$ for $20 \mathrm{pb}^{-1}$ and is dominated by the uncertainty in the jet energy scale (15\%).

\section{Expectations in the Semi-leptonic Muon Channel $(\mu+$ jets $)$}

Top-pair signal events in the $\mu+$ jets channel are selected by a single muon trigger. Offline selections then required exactly one isolated muon with $20 \mathrm{GeV}$ in $\mathrm{p}_{\mathrm{T}}$ and $|\eta|<2.1$ and at least four jets with $30 \mathrm{GeV}$ in $\mathrm{p}_{\mathrm{T}}$ inside the tracker acceptance. For $20 \mathrm{pb}^{-1}$, the selection is expected to yield 320 signal and 171 background events. The dominant background is expected to be coming from $\mathrm{W}+$ jets events. The event composition is shown in Figure 2.

Two data-driven methods to estimate QCD backgrounds have been developed: $\mathrm{ABCD}$ method and isolation extrapolation method. The ABCD method uses two weakly-correlated variables (impact parameter significance of the muon track and muon isolation) which have separate signal and background regions). Both methods give estimates in agreement to the true value within uncertainty.

To extract the signal events, a template fit is performed. In addition to the M3 variable, two other variables have been studied: $\eta(\mu)$ and the invariant mass of

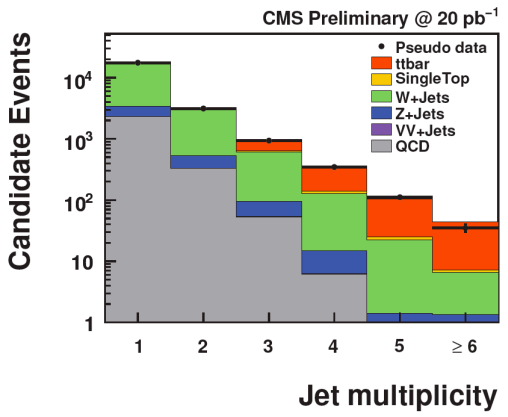

Figure 2: Event composition as a function of jet multiplicity for the $\mu+$ jets channel for $20 \mathrm{pb}^{-1}$. the hadronic top mass (M3') obtained from 3 -jets selected by minimizing a $\chi^{2}$ function. The statistical uncertainties are estimated to be $11.5 \%-18 \%$ for the three variables.

Sources of systematic uncertainty described in the previous section have also been studied in the muon channel. It is estimated that the total systematic uncertainty is $20-25 \%$, dominated by jet energy scale uncertainty.

An interesting new method [3] has been developed to estimate $\mathrm{W}+$ jets backgrounds by exploiting the charge asymmetry of the production of $\mathrm{W}$ bosons at the LHC. Studies show that given $100 \mathrm{pb}^{-1}$ of data a precision of $30 \%$ in the background estimation can be reached. Another analysis [4] employs boosted decision trees, a multivariate analysis technique, to measure the cross section. This study evaluated the statistical and systematic uncertainties of the measurement to be $8.6 \%$ and $25 \%$ respectively for $20 \mathrm{pb}^{-1}$.

\section{References}

[1] CMS Collaboration, The CMS experiment at the CERN LHC, JINST 3 S08004, 2008.

[2] CMS Collaboration, Plans for an early measurement of the t $\bar{t}$ cross section in the electron+jets channel at $\sqrt{s}=10 \mathrm{TeV}$, Physics Analysis Summary, TOP-09-004, 2009.

[3] CMS Collaboration, Prospects for the first measurement of the t $\bar{t}$ cross section in the muon-plus-jets channel at $\sqrt{s}=10$ TeV with the CMS detector, Physics Analysis Summary, TOP-09-003, 2009.

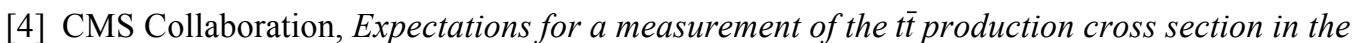
muon+jets final state using a multivariate technique, Physics Analysis Summary, TOP-09-010, 2009. 